Europhysics Letters

PREPRINT

\title{
First-order microcanonical transitions in finite mean-field models
}

\author{
Mickael Antoni ${ }^{1}$, Stefano Ruffo ${ }^{2}$ and Alessandro Torcini ${ }^{3,2,1}$ \\ 1 UMR-CNRS 6171 - Université d'Aix-Marseille III - Av. Esc. Normandie-Niemen, \\ 13397 Marseille Cedex 20, France. \\ 2 Dipartimento d'Energetica "S. Stecco" and CSDC, Università di Firenze, and INFN \\ and INFM, via S. Marta 3, 50139 Firenze, Italy. \\ 3 Istituto Nazionale d'Ottica Applicata, Largo E. Fermi 6, 50125 Firenze, Italy.
}

PACS. 05.70.Fh - Phase transitions: general studies.

PACS. 05.70.Ln - Nonequilibrium and irreversible thermodynamics.

PACS. 05.40.-a - Fluctuation phenomena, random processes, noise, and Brownian motion.

\begin{abstract}
A microcanonical first order transition, connecting a clustered to a homogeneous phase, is studied from both the thermodynamic and dynamical point of view for a $N$-body Hamiltonian system with infinite-range couplings. In the microcanonical ensemble specific heat can be negative, but besides that, a microcanonical first order transition displays a temperature discontinuity as the energy is varied continuously (a dual phenomenon to the latent heat in the canonical ensemble). In the transition region, the entropy per particle exhibits, as a function of the order parameter, two relative maxima separated by a minimum. The relaxation of the metastable state is shown to be ruled by an activation process induced by intrinsic finite $N$ fluctuations. In particular, numerical evidences are given that the escape time diverges exponentially with $N$, with a growth rate given by the entropy barrier.
\end{abstract}

Introduction. - There has been recently a renewed interest for systems with long-range interactions [1]. Phase transitions from clustered to homogeneous phases, occurring in simple models of globally coupled particles, have been analysed within different statistical ensembles $[1,2]$. It has been shown that, near transitions that are of the first order in the canonical ensemble, ensembles are inequivalent $[3,4]$. In the microcanonical ensemble the phase coexistence region can display a negative specific heat, corresponding to a convex entropy as a function of energy. In this region, entropy can be either a continuous and infinitely differentiable function of the energy (in the case of a continuous microcanonical transition) or it can display a discontinuity already in the first derivative. This case is denoted as microcanonical first order transition and is characterized by a jump in temperature as the energy is varied continuously $[3,5]\left({ }^{1}\right)$.

This latter case remains to be fully understood from the physical point of view: what does the coexistence of two temperatures, at equilibrium, for a given energy mean ? In this

$\left({ }^{1}\right)$ Other, more complicated, situations have been rigorously classified in [6]

(C) EDP Sciences 
Letter we present a careful analysis of the microcanonical first order transition that appears in a toy model that describes the motion of particles in a two-dimensional bounded domain (a torus). The model has been first introduced in Ref. [7,8], where the analysis was limited to microcanonical continuous transitions.

In the first part of this Letter, we fully characterize the phase transition by studying the intricate dependence of temperature on energy (the so-called caloric curve) and the dependence of entropy on both the energy and the order parameter, discussing also finite $N$ effects.

In the second part we concentrate on a study of the relaxation dynamics from a metastable state for a finite number $N$ of particles. This process had been originally investigated by Griffiths et al. [9] within the canonical ensemble. In this pioneering paper the authors have shown that, for a Curie-Weiss Ising model in an external magnetic field, the relaxation time of the metastable states grows exponentially with $N$, the exponential growth rate being given by the free-energy barrier per spin $\left({ }^{2}\right)$. More recently, the influence on the relaxation dynamics of extrinsic (thermal) noise source and of the intrinsic noise source due to finite $N$-effects has been analysed for a $\phi^{4}$-model with long range interactions [11].

We are not aware of any similar study within the microcanonical ensemble, especially in a situation of ensemble inequivalence. Indeed, the out of equilibrium dynamical behaviour near a microcanonical continuous transition has been already examined as far as the relaxation from unstable states is concerned $[12,13]$. The relaxation times have been shown to be typically proportional to some power of $N$. In our analysis, since no extrinsic noise is present, we emphasize the role played by the intrinsic noise source originated by finite $N$ effects. These intrinsic fluctuations induce an exponential divergence with $N$ of the relaxation time, with a growth rate given by the entropy barrier per particle.

The model. - The model we consider is a classical $N$-body Hamiltonian system defined on a two-dimensional periodic cell. The inter-particle potential is infinite ranged and the particles are all identical and have unitary mass. The Hamiltonian of the model is $H_{a}=K+V_{a}$, where $K=\sum_{i=1}^{N}\left(p_{x, i}^{2}+p_{y, i}^{2}\right) / 2$ is the kinetic energy while the potential energy reads

$$
V_{a}=\frac{1}{2 N} \sum_{i, j=1}^{N}\left[2+a-\cos \left(x_{i}-x_{j}\right)-\cos \left(y_{i}-y_{j}\right)-a \cos \left(x_{i}-x_{j}\right) \cos \left(y_{i}-y_{j}\right)\right]
$$

where $\left.\left.\left.\left.\left(x_{i}, y_{i}\right) \in\right]-\pi: \pi\right] \times\right]-\pi: \pi\right]$ represents the coordinates of the $i$-particle, $\left(p_{x, i}, p_{y, i}\right)$ the conjugated momenta and $a$ is a parameter. The complete phase diagram of this model has been previously presented in Ref. [8]. We will limit here our analysis to the parameter value $a=2$, for which the system undergoes a first order microcanonical phase transition. The different phases are conveniently characterized by the vector order parameter $\vec{M}_{z}=$ $\left(\langle\cos (z)\rangle_{N},\langle\sin (z)\rangle_{N}\right)$, where $z=x$ or $y$ and \langle\rangle$_{N}$ indicates the average over all the particles. It can be shown that $\left|\vec{M}_{x}\right| \approx\left|\vec{M}_{y}\right|=M$ and $M$ can be thought as the magnetization of the infinite-range Heisenberg XY Hamiltonian (10). In the low energy $U=H_{a} / N$ clustered phase (CP) all particles are trapped in a single cluster and $M \neq 0$, whereas in the high energy homogeneous phase (HP) they are uniformly distributed in the cell and $M \approx O(1 / \sqrt{N})$. At the microcanonical transition energy, both the order parameter and temperature have a discontinuity.

We have analyzed model (11) from an analytical and a numerical point of view. Since the microcanonical and the canonical variational problems define the same critical states $[8,14]$, it is convenient to first solve the model in the canonical ensemble. Indeed, via standard

$\left({ }^{2}\right)$ For more recent developments see [10] 
saddle-point techniques, one obtains all absolute and relative extrema of the free energy as a function of the order parameter [8]. These are also extrema of the entropy, although their stability properties are different in the two ensembles. States that are unstable in the canonical ensemble may become stable in the microcanonical: this is the mechanism at the origin of the negative specific heat in the microcanocical ensemble $\left({ }^{3}\right)$. From the entropy extrema, we get all the thermodynamics of the model in the mean-field $(N \rightarrow \infty)$ limit, including caloric curves and entropy barriers, that we discuss in the next Section. As far as the numerics is concerned, we perform molecular dynamics simulations (of course at finite $N$ ) using an accurate fourth order symplectic integrator $\left({ }^{4}\right)$.

Thermodynamics. - Let us first briefly resume what is known [8], about the phase diagram. In the canonical ensemble, model (11) exhibits a continuous transition from the $\mathrm{CP}$ to the HP for small $a$ values (namely for $0 \leq a<2 / 5$ ), above the canonical tricritical point, $a=2 / 5$, the transition becomes discontinuous with a finite energy jump. In the microcanonical ensemble the transition is continuous till the microcanonical tricritical point, $a \sim 1.15$, is reached. For $a>1.15$ a first order transition with a temperature jump is observed.

We concentrate here our analysis to the specific parameter value $a=2$, for which the transition is discontinuous in both the canonical and the microcanonical ensemble. In the canonical ensemble, at the transition temperature $T_{c}=0.664$, one observes an energy jump from $U_{1}=1.896$ to $U_{2}=2.664$, corresponding to a release of a latent heat. In this energy range the microcanonical ensemble gives different predictions. The $\mathrm{CP}$ is stable in the interval $\left[U_{1}, U_{c}=2.548\right]$, while in the interval $\left[U_{c}, U_{2}\right]$ the stable phase is the HP. The two phases are connected by a finite temperature jump at $U_{c}$ from $T_{C P}=0.618$ to $T_{H P}=0.548$. These results are confirmed by the numerical simulations (see Fig. 1(a)), although the sharp jump is smoothed by finite $N$ effects. The specific heat is negative from $U=2.240$ till $U_{B}=2.555$ (point B in Fig. 1(a)), but before reaching this energy value, at point A, the CP becomes metastable and remains such from A to B. At point B the specific heat vanishes and the phase turns to unstable, with an associated positive specific heat $\left({ }^{5}\right)$. The unstable curve BC (dot-dashed line in Fig 1(a)) joins the $\mathrm{CP}$ to the HP phase $(T=U-2)$. HP is metastable along CD in Fig 1(a), while for $U>U_{c}$ it is always stable.

The microcanonical transition can be better understood by considering the behaviour of the entropy per particle $s$ as a function of the order parameter $M$. In the energy range $\left[U_{C}, U_{B}\right]$ the entropy always exhibits two maxima separated by a minimum. The two maxima correspond to the $\mathrm{HP}\left(s_{H P}\right)$ and the $\mathrm{CP}\left(s_{C P}\right)$, respectively, while the minimum refers to an unstable clustered phase $\left(s_{\text {unst }}\right)$. Plotting the values of these extrema as a function of $U$, three distinct branches can be drawn (see Fig. 11 (b)): a concave one corresponding to the $C P$, and two convex ones corresponding to the $H P$ and to the unstable phase, respectively. For $U<U_{C}$ the entropy $s=s(M)$ exhibits a single maximum $s_{C P}$. At $U_{C}$, a second lower maximum $s_{H P}$ emerges together with a minimum, via a saddle-node bifurcation. At higher energies the height of $s_{H P}$ increases, while that of $s_{C P}$ decreases. At $U_{c}$ the two maxima reach the same height: this signals the first order transition energy. This is illustrated in Fig. 1(c), where $U_{c}$ is identified by the intersection of the barrier heights $\Delta s_{C P}=s_{C P}-s_{\text {unst }}$ and $\Delta s_{H P}=s_{H P}-s_{\text {unst }}$. For $U>U_{c}$ the $\mathrm{HP}$ maximum prevails and the $\mathrm{CP}$ phase becomes metastable. At $U_{B}$ the entropy minimum and the lower maximum corresponding to the $\mathrm{CP}$ merge and disappear via an inverse saddle-node bifurcation. Above such energy only one

$\left({ }^{3}\right)$ The first thorough discussion of the stability of microcanonical critical states was performed in Ref. [15] for self-gravitating systems

(4)More details on the integration method can be found in [7]

$\left({ }^{5}\right)$ Point B is a Poincaré turning point similar to that one investigated in Ref. [16] for isothermal spheres. 

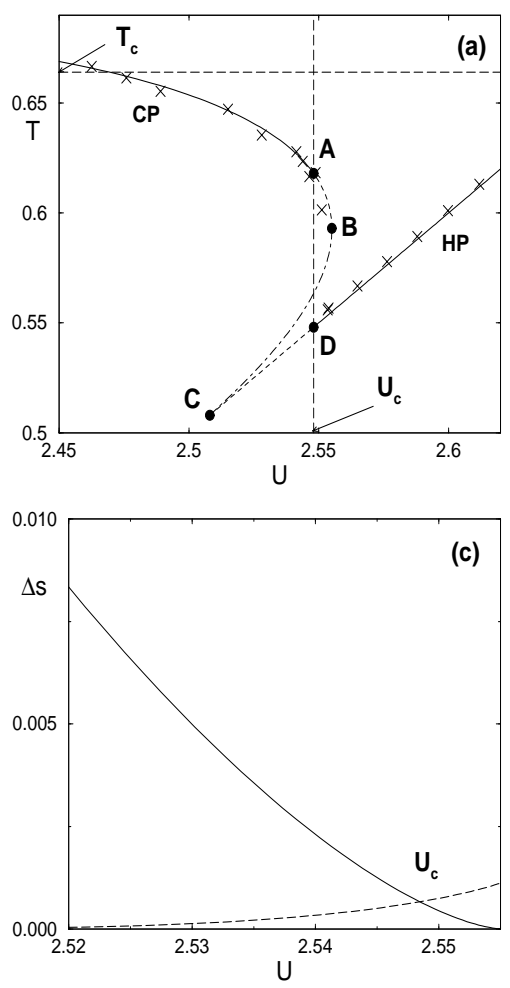
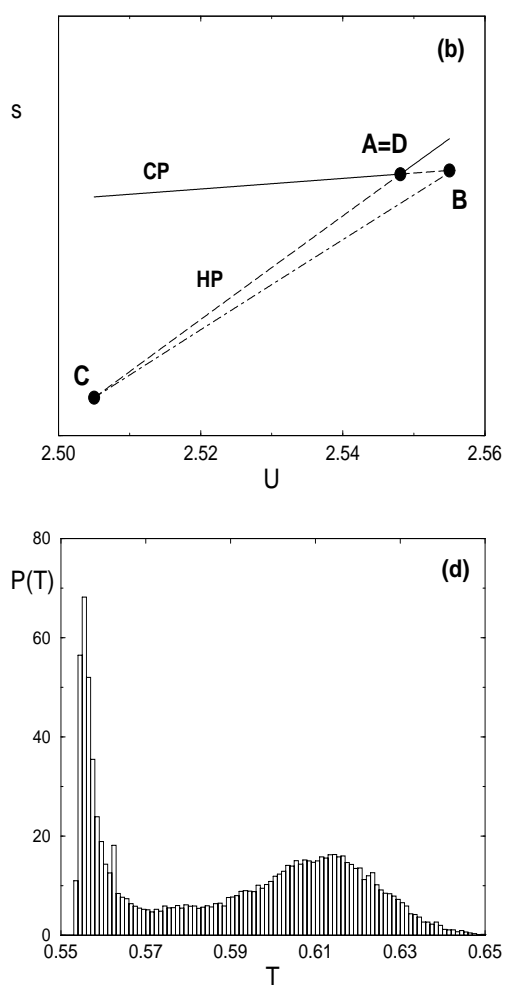

Fig. 1 - (a) Temperature $T$ as a function of energy per particle $U$ (caloric curve). Crosses refer to molecular dynamics simulations, where temperature is measured by the time average of the kinetic energy, while lines indicate mean-field analytical results. Solid lines refer to stable phases, dashed to metastable and dot-dashed to unstable. Numerical data have been obtained for $N=10,000$ by integrating the equations of motion of Hamiltonian (1) for a time ranging from 700, 000 to 900,000 typical periods of the motion. The vertical (resp. horizontal) dashed line corresponds to the microcanonical (resp. canonical) transition energy $U_{c}=2.548$ (resp. temperature $T_{c}=0.664$ ). (b) Sketch of the entropy per particle $s$ as a function of energy $U$. Solid lines refers to stable phases, dashed and dot-dashed to metastable and unstable, respectively. (c) Entropy barriers per particle versus energy $U$. The solid line refers to $\Delta s_{C P}$ and the dashed line to $\Delta s_{H P}$. The lines cross at the microcanonical transition energy $U_{c}$. (d) Histogram of the instantaneous temperatures close to $U_{c}(U=2.550)$ for $N=4,000$. Temperatures are measured by partially averaging kinetic energy over a time interval $\Delta t=300$. (The duration of the complete run is 900,000 proper periods.)

maximum in the entropy is present and it is associated to the HP.

Another strong indication that the transition is first-order comes from the fact that near the transition energy an intermittent behaviour is observed: the system jumps erratically from the $\mathrm{CP}$ to the HP and back $\left({ }^{6}\right)$. In Fig. 1(d) the histogram of the instantaneous temperatures is reported for a system of $N=4,000$ particles at an energy, $U=2.550$, close to $U_{c}$. Two peaks are present in the histogram: one is associated to the HP $\left(T \approx T_{H P}=0.550\right)$ and the other to the $\mathrm{CP}\left(T \approx T_{C P}=0.614\right)$. Hence, there is no mystery in the coexistence of two temperatures in equilibrium: temperature, in the microcanonical ensemble, is a derived quantity and is subject to fluctuations. The bimodal shape of the temperature histogram

$\left({ }^{6}\right)$ In the mean-field limit this intermittency is peculiar of the transition energy $U_{c}$ 
reflects the analogous bimodality of the order parameter fluctuations, which is a consequence of the first order nature of the phase transition. Similarly, in a canonical simulation, one would observe a bimodal energy distribution, with peaks at $U_{1}$ and $U_{2}$.
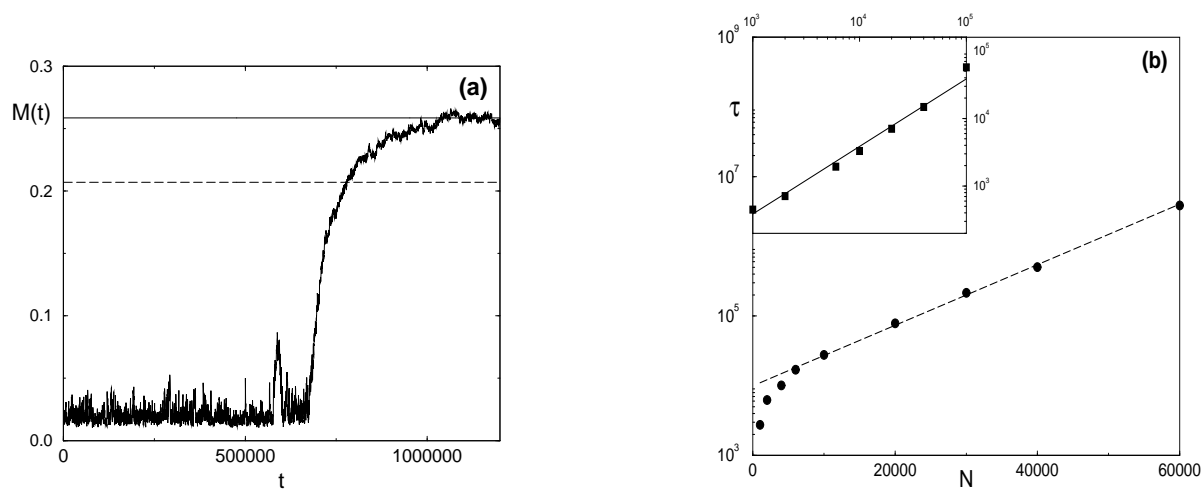

Fig. 2 - (a) Istantaneous magnetization $M(t)$ versus time. $M(t)$ has been obtained by averaging over short time windows of duration 300 and over 20 initial conditions. The asymptotic magnetization value is $M=0.2586$ (solid line) and the dashed line indicates the threshold value used for the determination of the lifetime (i.e. 0.207). The data refer to $U=2.530$ and $N=4,000$. (b) Lifetimes $\tau$ of the metastable state HP as a function of $N$ in a log-lin scale. Circles refer to numerical data at $U=2.530$, the dashed line to the best fit obtained in the interval $6,000<N<60,000$ (see Table I). In the inset we report numerical results (squares) for $U=2.40$ in a log-log scale, the solid line is a linear fit to the data with slope one. The data have been obtained by averaging over 10 to 1,000 initial conditions.

Relaxation Dynamics. - We are interested in characterizing the differences in the relaxation dynamics from an unstable state towards a stable one, with respect to an initial metastable state. As detailed above, an unstable state is an entropy minimum, while a metastable state is a relative maximum, separated from the stable state by an entropy barrier.

It is reasonable to conjecture that in the first case the relaxation process is dominated by a "diffusive like" behaviour, while in the second situation the activation across an entropy barrier should play a fundamental role. At variance with canonical ensemble dynamics [11], the activation process can be induced only by finite $N$ fluctuations, since there is no coupling to an external bath. Hence, we expect that the relaxation time from an unstable state will typically increase with a power law in the particle number, the relaxation process being dominated by the granularity of the system, that drives it to equilibrium through collisional effects of the type encountered in self-gravitating systems [17]). Instead, for a metastable state, we should find a noticeably different behaviour. Let us consider the situation where the CP is stable, while the HP is metastable. By following the standard fluctuation theory [18], we expect that the probability to observe a given value of the magnetization in the interval $[M: M+d M]$ is given by

$$
w(M) d M=\text { const. } \mathrm{e}^{\left[S(M)-S_{H P}\right]} d M=\left(2 \pi<M^{2}>\right)^{-\frac{1}{2}} \mathrm{e}^{-\frac{M^{2}}{2<M^{2}>}} d M
$$

where $S(M)=N s(M)$ is the entropy as a function of the order parameter, $S_{H P}=N s_{H P}$ is the entropy of the HP (for which $M=0$ ) and $<M^{2}>\sim O(1 / N)$. As soon as the magnetization reaches the value corresponding to the entropy minimum $M_{u n s t}$, the system is quickly driven 
towards the CP. Therefore, the relaxation time from a metastable state should diverge as :

$$
\tau \propto\left[w\left(M_{\text {unst }}\right)\right]^{-1} \propto \mathrm{e}^{\left[s_{H P}-s_{\text {unst }}\right] N}
$$

and we will observe an activated escape process as long as $\Delta s_{H P} \geq 1 / N$.

Let us check these two distinct relaxation behaviours in numerical experiments. In order to measures the lifetime of a metastable (unstable) HP, the system is initialized with zero magnetization (indeed due to finite $N$ effects $\langle M>\sim 1 / \sqrt{N})\left({ }^{7}\right)$. Once the initial state is prepared, we follow its time evolution by monitoring magnetization. A typical behaviour is reported in Fig. 2(a). Then, we register the time $M$ needs to reach $80 \%$ of its asymptotic value. The lifetime of the HP state is finally determined by averaging over several different initial conditions.

We first examine the relaxation of a metastable state, therefore the system is initialized with an energy in the range $\left[U_{C}, U_{c}\right]$. In this interval entropy exhibits an absolute maximum $s_{C P}$ (corresponding to the $\mathrm{CP}$ ) and a relative maximum $s_{H P}$ (corresponding to the HP) separated by a minimum $s_{\text {unst }}$. If we prepare the initial state in the HP this will have a lifetime $\tau$ that we expect to grow as $\exp \left[\Delta s_{H P} N\right]$. In particular, as shown in Fig $2(\mathrm{~b})$ at $U=2.530$, for $N>6,000 \tau$ has indeed an exponential growth with $N$. Moreover, as reported in Table \the numerical estimation of the growth rates $\Delta s_{n u m}$ at three different energies in the interval $\left[U_{C}, U_{c}\right]$ are in agreement with the mean-field value $\Delta s_{H P}$. As a final remark, one should notice that for small $N$ (e.g. for $100<N \leq 6,000$ at $U=2.530$ ) the escape time grows proportionally to $N$ instead of exhibiting an exponential growth (as it can be seen also in Fig[2(b)). This behaviour resembles the relaxation from an unstable state and it is due to the fact that for such small number of particles $1 / N>>\Delta s_{H P}$. Therefore the magnetization fluctuations are so large to easily overcome the entropy barrier, that does not play any role here. In order to measure the lifetime of an unstable HP, we take the energy $U=2.4$, for which the zero magnetization state is unstable and the CP is stable. At $U=2.4$ the transition times $\tau$ scale clearly with $N$ as shown in the inset of Fig. 2(b). Such type of divergence was first observed in Refs. [12] in model (11) for $a=0$. Recently, Yamaguchi [13] has studied the same model, finding that $\tau$ diverges as $N^{1.7}$. On the other hand Bouchet [19] has shown, again for $a=0$, that the single particle self-diffusion time increases linearly with $N$.

TABLE I - Entropy barries $\Delta s_{H P}$, as estimated from the mean-field microcanonical analytical results and by direct molecular dynamics simulations. The escape times from the homogeneous metastable phase are evaluated by averaging over 10 to 1,000 initial conditions. The numerical values for $\Delta s$ are estimated by fitting the escaping times as a function of $N$, tipically in the interval 4,000 - 60,000.

$\begin{array}{lcr}U & \Delta s_{H P} & \Delta s_{\text {num }} \\ 2.530 & 1.28 \times 10^{-4} & (1.0 \pm 0.1) \times 10^{-4} \\ 2.540 & 3.33 \times 10^{-4} & (3.4 \pm 0.5) \times 10^{-4} \\ 2.545 & 5.00 \times 10^{-4} & (5.8 \pm 0.4) \times 10^{-4}\end{array}$

Concluding remarks. - In this Letter we have examined a first-order microcanonical transition from a clustered to a homogeneous phase for a $N$-particle model with infiniterange interactions. Even in the mean-field limit canonical and microcanonical ensembles are

$\left({ }^{7}\right)$ The initial configurations have been realized in two different ways, but significant discrepancies have never been observed. In one case the particle positions have been chosen randomly within the cell and the velocities accordingly to the Maxwell-Boltzmann distribution. In the other one an equilibrated configuration with energy $U>U_{c}$ (where the HP is stable) is taken and the velocities are rescaled to obtain the desired energy. 
inequivalent. While in the canonical ensemble the transition exhibits an energy jump at the transition temperature, in the microcanonical a temperature jump is observed at the transition energy. Moreover, specific heat is negative in a wide energy range. We have elucidated the intricate structure of stable, metastable and unstable phases that coexist near the transition.

A more physical grasp is obtained by considering the entropy of the system as a function of the order parameter. In a large energy domain, we observe the coexistence of a stable and a metastable phase, corresponding to the absolute and relative entropy maximum, respectively. In this energy interval the two maxima are separated by an entropy minimum, associated to an unstable state. We have shown that the relaxation from the metastable state is due, for sufficiently large $N$, to an activated crossing of an entropic barrier and that the activation mechanism is induced by finite $N$ intrinsic fluctuations. The escape time from the local entropy maximum grows exponentially with $N$, and the growth rate is given by the entropy barrier per particle. We have further checked that, instead, the relaxation time from an unstable state grows linearly with $N$.

These results represent a first step towards the assessment of a more rigorous theory of the dynamical evolution of a microcanonical system with long-range interactions trapped in a metastable state. A further refinement will require the derivation of an appropriate FokkerPlanck equation describing out of equilibrium fluctuations.

$$
* * *
$$

We acknowledge discussions with J. Barré, F. Bouchet, E.G.D. Cohen, T. Dauxois, D.H. Gross and D. Mukamel. This work has been partially supported by the MIUR (Italy) under COFIN03.

\section{REFERENCES}

[1] Dynamics and Thermodynamics of Systems with Long Range Interactions, edited by DAuXOIS T. et al., Vol. 602 (Lecture Notes in Physics, Springer, Berlin) 2002.

[2] Gross D.H.E., Microcanonical thermodynamics:Phase transitions in "small" systems (World Scientific, Singapore) 2000.

[3] Barré J., Mukamel D. and Ruffo S., Phys. Rev. Lett., 87 (2001) 030601.

[4] Ispolatov I. and Cohen E.G.D., Physica A, 295 (2001) 475.

[5] Chavanis P.H. and Ispolatov I., Phys. Rev. E, 66 (2002) 036109.

[6] Barré J., PhD Thesis, ENS-Lyon (2003) .

[7] Antoni M. and Torcini A., Phys. Rev. E, 57 (1998) R6233; Torcini A. and Antoni M., Phys. Rev. E, 59 (1999) 2746.

[8] Antoni M., Ruffo S., and Torcini A., Phys. Rev. E, 66 (2002) R025103.

[9] Griffiths R.B., Weng C-Y , and Langer J.S., Phys. Rev., 149 (1966) 301.

[10] Paul W., Heermann D.W., and Binder K., J. Phys. A: Math. Gen., 22 (1989) 3325.

[11] Rostiashvyyili V.G. and Schilling R., Z. Phys. B, 102 (1997) 117.

[12] Antoni M. and Ruffo S., Phys. Rev. E, 52 (1995) 2361; Antoni M., Hinrichsen H. and Ruffo S., Chaos, Solitons 8 Fractals, 13 (2002) 393.

[13] Yamaguchi Y.Y., Phys. Rev. E, 68 (2003) 066210.

[14] Barré J., Ruffo S. and Mukamel D., in [1].

[15] Katz J., Mon. Not. R. Astron. Soc., 183 (1978) 765.

[16] Katz J. and Окамото I., Mon. Not. R. Astron. Soc., 317 (2000) 163.

[17] Binney J. and Tremaine S., Galactic Dynamics (Princeton Univ. Press, Princeton) 1987.

[18] Landau L.D. and Lifshitz E.M., Statistical Physics (Pergamon Press, Oxford) 1985.

[19] Bouchet F., preprint (cond-mat/030517), (2003). 